\title{
The Research on Campus Panoramic System Based on VR Technology
}

\author{
Zhu Yafen*, Jiao Baocong, Pang Xin \\ Department of Educational Technology, Capital Normal University, Beijing, China \\ Email address: \\ 18370997808@63.com (Zhu Yafen),jiaobc3093@126.com (Jiao Baocong), pangxin163163@163.com (Pang Xin) \\ *Corresponding author
}

To cite this article:

Zhu Yafen, Pang Xin, Jiao Baocong. The research on Campus Panoramic System Based on VR Technology. Science Journal of Education. Vol. 6, No. 2, 2018, pp. 50-54. doi: 10.11648/j.sjedu.20180602.12

Received: January 18, 2018; Accepted: March 21, 2018; Published: May 11, 2018

\begin{abstract}
With the constant development of social productive forces and science and technology, the demand for VR technology in various industries is growing day by day. More and more attention will be paid to the research of VR technology. However, the current research does not present a complete system of introducing virtual reality technology into the campus. Therefore, in this paper, image synthesis method was used to Capital Normal University, for example, to design and develop a (campus panoramic system) based on virtual reality campus panorama system so as to construct a complete system for others to learn from. The system can not only support computer-side transmission, but also it can spread the convenience of support for two-dimensional code push through the mobile terminal to bring intelligent navigation to users. Moreover, through seamless connection with VR glasses, immersive feeling can be enjoyed even though the users are not in it. In addition, it supports interactive testimonials. During the establishment of the system, UAV and GoPro and other related hardware is used for pre-material acquisition, Ptgui software is used for later panorama synthesis, as well as the Photoshop software which is used to make appropriate changes to the picture and color, and finally the works are uploaded to 720 cloud platform for dissemination and use. The system provides the overall ideas and steps to make a campus panoramic system for others to learn.
\end{abstract}

Keywords: Virtual Reality, Campus Panorama System, Panorama, Ptgui, 720 Clouds

\section{Introduction}

With the continuous development of social productive forces and science and technology, the demand for VR technology in various industries is increasing day by day. Growing attention are paid to the research of VR technology. As the ninth largest art of mankind [1], it is introduced into the study of campus panoramic system In the virtual reality is the inevitable trend of development. However, the present research does not present a complete system of how to introduce virtual reality technology into the campus. In this paper, the image synthesis method was used to take the Capital Normal University as an example to design and develop a campus panoramic system based on virtual reality in order to construct a complete system for others to learn. The campus system features smart navigation, seamless connectivity with VR glasses, interactive testimony with other users, the freedom to switch between arbitrary scenes, and the ease with which two-dimensional code can be transmitted. The system was established from the early collection of material, post-processing of materials to upload platform and the spread of virtual campus systems and other aspects of elaborate. The system not only allows students to improve their relationships with others, but also allows more people to learn about the school's campus environment and culture online anytime and anywhere they are.

\section{Related Theory}

\subsection{Virtual Reality}

Virtual Reality (VR), also translated as Spiritual Environment Technology, originated in the 60 s of the last century and is the result of scientific and technological progress since the 20th century. In a virtual environment, 
users interact directly with objects in a virtual reality scene to sense the world's immersive feelings through sensory stimuli such as sight, hearing, touch, and the like [2] It mainly embodies computer technology, computer graphics, sensor technology, ergonomics, human Machine interaction theory and other areas of the latest achievements. [3] Techniques for users to interact and interact with objects in the virtual world in a natural way with the necessary equipment to create immersive experiences and experiences. Virtual reality is a kind of scientific method and science and technology that mankind creates and forms during the process of exploring nature and knowing nature. It gradually forms a kind of scientific method and science and technology for understanding nature and simulating nature so as to better adapt and use nature. [4]

Burdea \& Coiffet (1992) summarized the important features of virtual reality as "3I," namely, Immersion, Interaction, and Imagination. [5]

1) Immersion: Reality, immersive feeling. The user interacts naturally with the virtual world by means of special input / output devices such as VR glasses. The virtual reality technology provides users with sensory simulations such as visual, auditory and tactile sensations which enable the user to devote himself into this virtual world in all aspects, this is the primary feature of virtual reality.

2) Interactivity: refers to the virtual environment, the user's input to respond, and on this basis, by the user's influence or to influence the user. Users manipulate objects within the virtual environment as they are in a real environment. For example, users can truly feel the realism of the environment and the weight of the object.

3) Imagination: also refers to the creative, refers to the process of user interaction in the virtual environment, through imagination, reasoning logic and judgments and other thinking process, the ability to imagine the future.

\subsection{Campus Panorama System}

At present, the construction of virtual campus has shifted from the traditional forms of text, pictures and two-dimensional maps to three-dimensional virtual campus.[6] In this paper, the method of panoramic stitching was used to design and develop campus panoramic system, which means making a series of panoramic to establish campus panoramic image system. The so-called panorama refers to the image that has a wide field of view (FOV, Wide Field of View); and the so-called splicing refers to the integration of multiple single image into an image. Specifically, panoramic stitching is to use multiple cameras to shoot the same scene at different angles, and then to correct, denoise, match and blend the multiple images obtained, and finally to build images which exhibit high quality, clear, smooth edges and high resolution. [7]

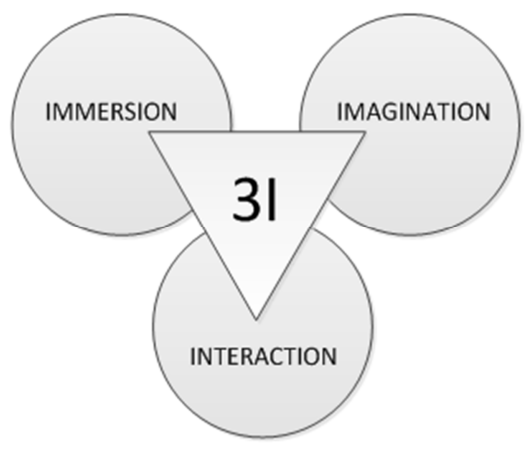

Figure 1. 3 I virtual reality features.

\section{Campus Panorama System Design}

\subsection{System Framework Design}

Campus panorama system mainly to achieve two major functions: physical campus "virtualization", imagination campus "reality" [8] The design of campus panorama system in this paper is based on the internal structure of campus. It can be divided into three parts according to the function of its internal area: learning area, amusement park and living service area. In addition, as a campus panoramic system, a panorama Aerial view, a glance around the school environment can be seen, therefore, this article will be divided into four parts of the campus panorama to design and development. as shown in Figure 2.

Then based on these four major parts, the division of the various districts of the campus is clear. The learning area provides a place for learning. It generally consists of a teaching building, a library, an academic lecture hall, and the like. Recreational diocese, which is places for entertainment and leisure to learn, generally includes: museums, auditoriums, activity centers, cultural institutes. Living service areas that which is places for diet, living and office space, summarized include: canteens, playgrounds, dormitories, gardens, streets and financial services. Aerial view of the campus is the overall panorama exactly. UAVs and other related hardware was used to collect pre-material in the central school in the acquisition process. According to the campus panorama system framework, each area of the school will be assigned until all the regions are divided. The material collection point will be determined before, which makes it convenient to film material purposefully. 


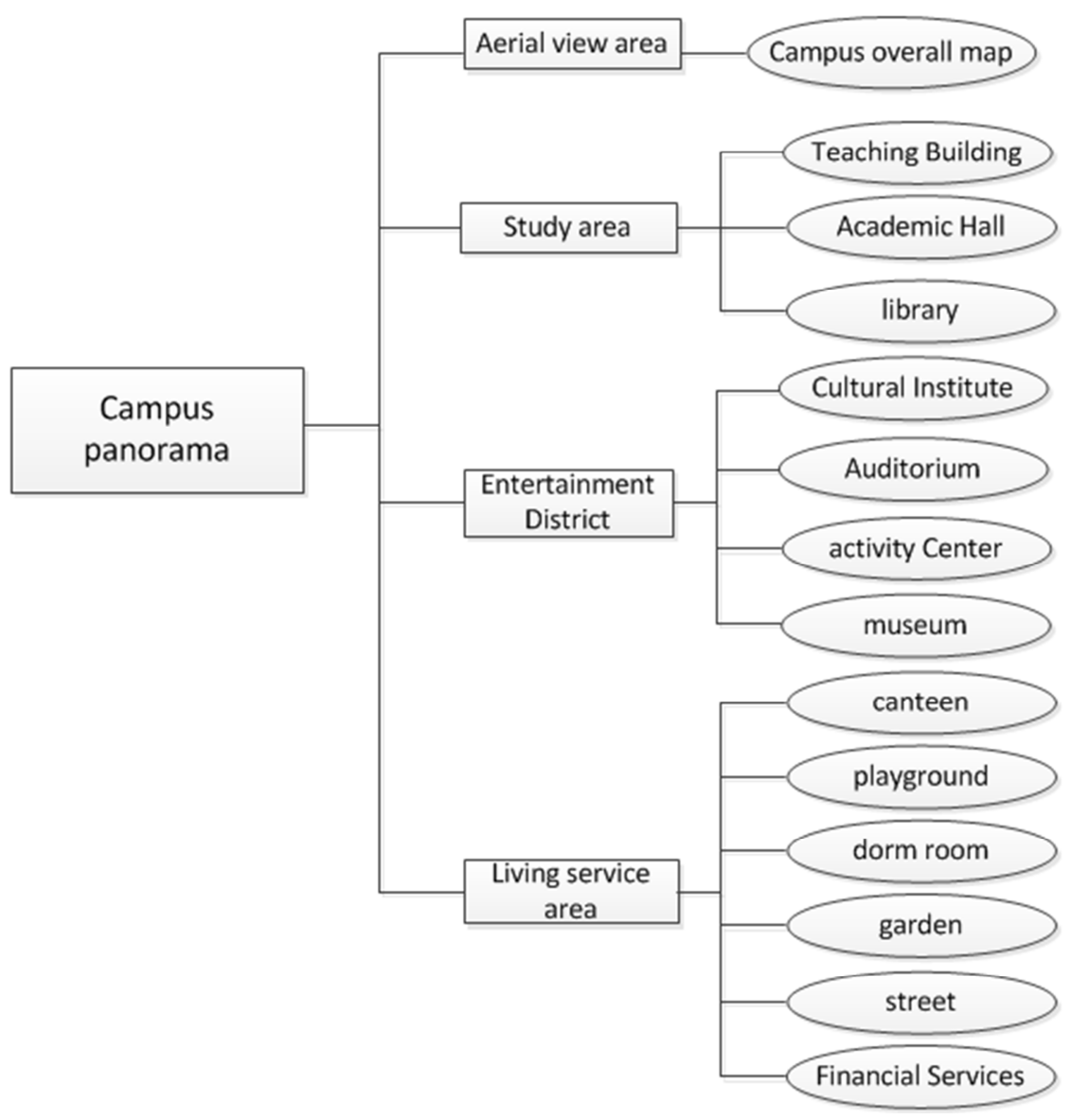

Figure 2. Campus panorama system framework.

\subsection{System Function Design}

(1) smart navigation

The campus panorama system presents all of the campus's geographical locations and shows an overview of the campus distribution. When using the campus panorama system, it can not only help users to know where they are, but also it can help to find out where they need to go to reach their destination through navigation buttons. There is a text description that shows where to go for navigation buttons, users can click on the corresponding navigation button according to their needs.

(2) Seamless connection with VR equipment

Under normal circumstances, a certain platform is needed to make it possible for the panorama to be played, 2VR software can be used for offline playback, but for online play the web platform are used normally, for example: 720 cloud, sky city. But the play is similar to the flat graphics, while with the combination using of the VR device and the system, the two-dimensional panorama can be changed into three-dimensional. The three-dimensional effect makes it possible for to the user to enjoy immersive feeling even though they are not in it.

(3) interactive speech

The campus is home to many students and contains many memories of their own. After leaving the campus, rare opportunity will be obtained for them to go home like the student days. Throughout the campus panoramic system, ideas of "going home and having a look " can be easily achieved. Based on this platform, a communication platform was built for students, in which the ideas can be published, moreover, other partners can also deliver their interactive feedback.

(4) Scene freely switch

In the campus panoramic system, there is scene switch button in the bottom row, through which the scene can be freely switched to places users want to see. In addition, there are scenes in the screen navigation buttons, the scene navigation button can also be clicked to switch to places users want to see. During the process of looking at one of the scenes, it can not only be dragged through the mouse for 360-degree implementation of this scene without dead ends, but also it can be zoomed in and out through the wheel switch of the mouse.

\section{(5) Spread easily}

Campus panorama system can not only be displayed on the web, but also it supports the mobile terminal playback. In addition, in the 720 platform, panoramic system can be converted into the form of two-dimensional code to spread. Figure 3 shows the two-dimensional code of the Panorama System of Capital Normal University, which can make it able to view the whole picture of Capital Normal University immediately after picking up the phone and scanning the code. QR codes can also be shared directly to others for 
viewing and sharing by others.

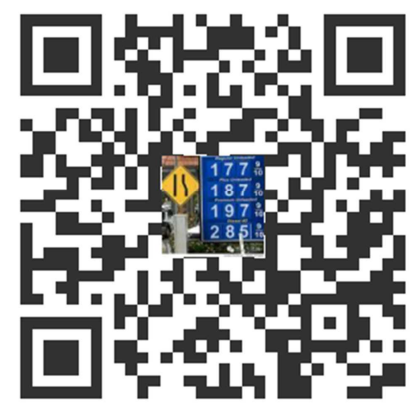

Figure 3. Capital Normal University panoramic system two-dimensional code.

\section{Realization of Campus Panorama System}

(1) Access to panoramic material

According to the design of the framework of the campus panorama system, materials were collected in turn inside the campus, and the GoPro's panoramic shooting function was used to build six machines to implement the acquisition of panoramic materials without dead ends. In order to make the collection of materials more smooth, attention were needed to pay to the following four points. First, the light intensity. During the process of material acquisition, it needs to choose the right light, sufficient light intensity circumstances to implement, in order to avoid filming photos overexposed or underexposure. Second, appropriate location selected, since the purpose of this filming is 360 degrees without dead shooting, it is necessary to select the location as far as possible to make the collection of six machines can be collected on average. Generally, it is more appropriate to select the scene location of the mid-point. Third, it is important to protect the safety of electronic equipment. For electronic equipment bringing in and out, the first is to protect its security, for example, it is unsuitable for outdoor shooting in rainy days in order to avoid equipment damage caused by rain. What's more, there is a professional shipping box for the use of carrying machine and equipment to avoid unnecessary collision. Fourth, the file is stored clearly. After the completion of shooting, the folder is divided into many categories according to the location name of machine so as to facilitate the smooth progress of post-splicing.

(2) Panoramic mosaic

After the completion of the acquisition, in practice, there are lots of software available today that can produce and stitch these panoramas, such as Pixtra PanoStitcher, PanoramaStudio, Pano2QTVR and etc. Among them, Ptgui software is a small as well as refined panorama synthesis software. Ptgui software is used for panorama splicing [9]. The main function of Ptgui is to splice the collected photos. After starting Ptgui, the first step is to load the images to be spliced, and then select the scenes one by one from the newly stored materials. The second step is to align the image and then select "Align Image". In some cases, due to few reference objects between adjacent images, it is hard for the software to find enough control point, which results in failing to synthesize panorama. Therefore, it is necessary to manually add the control point and optimize until the successful splicing panorama can be synthesized, and finally click to create a panorama. The third step is to save. Modify the path and file name (the default path for the original photo path) in the "output file ", click the" Create Panorama "in the lower left corner after setting up, and then it is saved.

(3) Modify and color

Images from the Ptgui software are sometimes less than satisfactory, for example, when a shadowy or unwanted person appears in the scene, and in this time, it is needed to get rid of it. Photoshop is a professional, advanced image analysis and processing software, it is very powerful, with a variety of image adjustment capabilities, and it can draw and edit graphics, which makes it become one of the main image processing software. Advanced principles is used in the graphic processing and creation of various applications so that it achieves good results. [10] It is the best choice to use it to process the spliced pictures. Through the rational use of the masks, the pictures can be modified by using the repair tools or the imitation stamp tools. After the modification, the color can be adjusted according to individual needs, so that the whole scene presents your favorite style.

(4) Upload platform

After modifying the stitched picture, it is required to be uploaded to the platform for communication, such as 720 clouds, the sky city. And in this paper, the 720 cloud is mainly introduced. 720 cloud panorama VR community, is a one-stop solution platform for 360 panoramic photography, VR panoramic video capture, VR aerial panoramic aerial photography, 3D virtual reality production, panoramic upload, share, display, roaming, and creator interaction exchange, VR supply and demand trading. It can not only share works in the platform, but also users can launch forums to share their own flying experience with fellow friends. There are generally activities to promote the production of finished products by Feyou such as the annual photo contest and other events. After entering the platform, first login, and then click the button "upload", the modified mosaic map will be uploaded. Then enter the panorama of the editing page, in order to make the panorama more vivid, it is able to add roaming hot spots, sounds, text and so on in the panorama. A hot spot is equivalent to a hyperlink between two panoramas. Enter the appropriate page to modify according to its corresponding request, after modifying, then save and preview.

\section{(5) Test and share}

After saving the preview, the mobile terminal is used to test the generated two-dimension code, and the whole view of the campus panoramic system is checked from the mobile terminal. If there is a problem that needs to be modified, the platform may be re-entered to modify the corresponding problem. After the page of the mobile campus panorama system is determined that there is no problem, then the 
generated two-dimensional code can be saved as a picture for sharing with other users. The generated system link is as follow: http://720yun.com/t/cf926x8dxtn?pano_id=1171039

\section{Conclusion}

Panoramic equipment and panoramic software was comprehensively applied to design and develop the Capital Normal University campus panoramic system. Through the system, users can truly and intuitively understand the campus environment, intelligent navigation. Meantime, some interactive operation can be done according to their own needs. The system presents plenty of advantages, for example the construction cost is low, the method is simple, the method of communication is convenient, the interactivity is provided, and the panoramic image exhibits a good display degree and etc. Compared with other virtual reality technologies that require three-dimensional modeling and simulation technology, it only needs to splice the captured images, so that it enables people to realize immersive virtual roaming. The process of making Capital Normal University campus panorama system in this paper can provide a reference for other people who want to make the panorama. Panorama can be widely applied, for example, it is widely used in tourist attractions, real estate applications, digital city environment and so on. It is believed that in the near future, with the development of virtual reality technology, panorama technology will be more and more adapted to people's needs, and it will get better development.

\section{Acknowledgements}

This article is a key project of the "12th Five-Year" Plan of Education Science in Beijing, "Basic Education School Classroom Teaching Experimental Research" (ABA15008) Fund Project. The authors also would like to acknowledge the great support from the mentor, as well as my partner for the help during the implementation of the project.

\section{References}

[1] WANG Yan-an, ZENG Jun-feng, An Yun-hu a. Design of a virtual walk th rough system for cam pus of yang tze university [J]. Hubert Agri-cu ltura $1 \mathrm{~S}$ ciences, 2010, 49 (9): 2237-2240. WANG Yan-an, ZENG Jun-feng, AN Yun-hua. Construction of Virtual Tour System in Yangtze University Campus [J]. Hubei Agricultural Sciences, 2010, 49 (9): 2237-2240.

[2] ZHANG Shu-cai, WANG Ming-jun. Research on Courseware Production Based on Virtual Reality Technology [J]. Science and Technology Information, 2010 (21): 584-585.

[3] Huang Jianling, Zou Hui. Experimental teaching system based on virtual reality technology and its characteristics [J]. China Electrochemical Education, 2009, (04): 108-111. [2017-08-26].

[4] Zhao Qinping. Review of virtual reality [J]. Science in China (Series F: Information Science), 2009, 39 (01): 2-46. [2017-08-26].

[5] Liu Dejian, Liu Xiaolin, Zhang Yan, Lu Aifan, Huang Ronghuai. The Potential, Progress and Challenges of Virtual Reality Education [J]. Open Education Research, 2016, 22 (04): 25-31. -26]. DOI: 10.13966 / j.cnki.kfjyyj.2016.04.004.

[6] Wu Xuewen, Shi Zejie, Li Hui, Zhu Xiaoling. Design and Development of Virtual Campus System Based on PV3D and Flex [2017] ].

[7] Journal of Chongqing Technology and Business University (Natural Science Edition), 2012, 29 (12): 60-65 + 71. [6] JIANG Tie, ZHU Gui-bin, SUN AO. Review of the research status of panoramic image mosaic $[\mathrm{J}] 26$.

[8] WANG Li-Rong, ZHU Dong-Ming. Design of Teaching System Based on Virtual Reality [J]. China Distance Education, 2011 (01): 67-69.

[9] Gao Yi. Panoramic Photosynthesis Technology and the Use of PTGUI [J]. Computer Knowledge and Technology, 2010, 6 (25): 7114-7117.

[10] Cui Chunli. Application of Photoshop Software in Image Analysis [J]. Computer Development and Application, 2012, 25 (12): 83-85. [2017-08-27]. 